\title{
Relação das pessôas e instituições que doaram obras ou revistas á Biblioteca da Faculdade, no periodo compre- endido entre 16 de novembro de 1935 e 15 de Abril de 1936
}

A. F. Cesarino Junior

A. Pompêo

A. Viriato Pereira

Aben-Attar Netto

Agustin Venturino

Alberto J. Robbe

Alcantara Machado

Alcides Bezerra

Aleksander W. Matuszewski

Alexandre Corrêa

Alfredo Mendizabal Villalva

Alpheu Domingues

Antonio Constantino

Antonio de Padua Salles

Arthur Barreto de Aguiar

Associação dos Proprietarios de São Paulo

Biblioteca da Reitoria da Universidade de São Paulo

Biblioteca do Ministerio da Fazenda

Biblioteca Nacional de Buenos Aires

Biblioteca Riograndense

Cantinho Filho

Carlos Fernandes

Carlos Xavier Paes Barreto

Celso Vieira

Centro Paulista

Consulado do Japão

Demetrio Lemos
École Libre de Sciences Politiques

- Paris

Edgar Dehn

Editorial Reus, S. A. - Madrid

Elisa Novah

Enrico Altavilla

Ernesto de Souza Campos

Flaminio Favero

Flavio Mendes

Francisco C. Bendicente

Francisco Loffredo

Francisco Morato

Francisco Ribeiro dos Santos

Gennaro Escobedo

George H. Jaffin

Godofredo de Faria

Gremio de Cultura Mauá - Pelotas

Grupo "Amor y Vida" - Barcelona

Hans Kreller

Imprensa Oficial do Estado de São Paulo

Imprensa Oficial do Estado de do Ceará

Imprensa Oficial do Estado de do Maranhão

Imprensa Oficial do Estado de do Pará

J. Conangiola Fontanilles

J. M. de Azevedo Marques

Jamil Miguel Nami 
Joăo Arruda

João Rodrigues de Meréje

José Carlos de Macedo Soares

José de Moraes Leme

José Fernandes Moreno

José Gabriel Pinto Coelho

José Peco

Legación de Colombia en España

Leon Walther

Lins e Silva

Livraria Universal

Loreto Filho

M. J. Mac-Pherson

Manuel Augusto Vieira Netto

Manoel Pereira

Marcello Finzi

Ministerio das Relações Exteriores

- Rio de Janeiro

Ministerio das Relações Exteriores do Panamá

Ministerio do Interior - BuenosAires

Noé Azevedo

Oficinas Graficas - Santuario d'Aparecida

Orlando Gomes

Oscar Correia Pina
Paulo Alberto

Paulo de Macedo Couto

Plinio Ayrosa

Prefeitura Municipal de São Bernardo

Procuradoria Judicial da Fazenda. do Estado

R. Oliva

Regio Consolato Generale d'Italia.

Renato Kehl

Rodrigo Octavio

Rodrigues Doria

Rubio Braziliano

Rui Calasans

Secretaria da Camara dos Deputados Federal

Secretaria da Educação e Saúde Publica

Secretaria da Justiça e Segurança: Publica

Secretaria da Viação e Obras Publicas do Estado de São Paulo. Tarquinio Giglio

Tomas Liscano

Universidad de Breslau

Vieira Ferreira

Waldemar Ferreira 